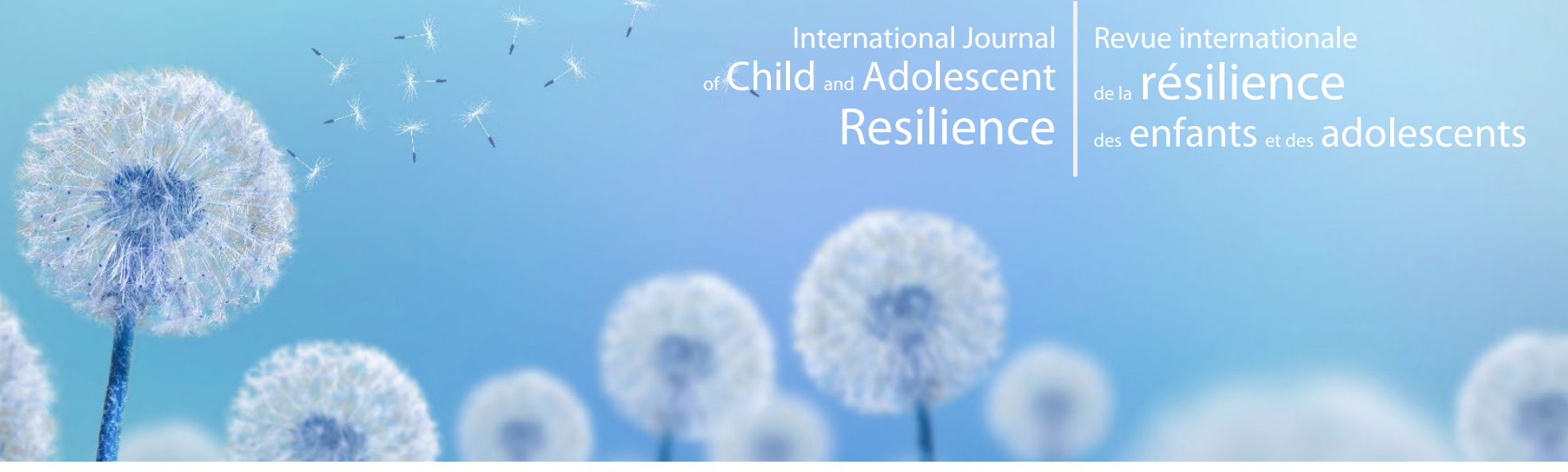

\title{
Prévenir les violences sexuelles en milieu autochtone : Retour sur la formation au Programme Lanterne|Awacic
}

\section{Virginie ATTARD ${ }^{1}$, Jacinthe DION ${ }^{2}$, Christiane BERGERON-LECLERC'1, Vincent TREMBLAY ${ }^{3}$, Martine HÉBERT ${ }^{4}$ et Mireille DE LA SABLONNIĖRE-GRIFFIN²}

\footnotetext{
Département sciences humaines et sociales, Université du Québec à Chicoutimi, Chicoutimi, Québec

2 Département des sciences de la santé, Université du Québec à Chicoutimi, Chicoutimi, Québec

3 Module d'informatique et de Mathématiques, Université du Québec à Chicoutimi, Chicoutimi, Québec

4 Département en sexologie, Université du Québec à Montréal, Montréal, Québec
}

Correspondance : Virginie Attard, M.T.S (c), Département des sciences humaines et sociales, Université du Québec à Chicoutimi, 555, boul. de I'Université, Chicoutimi, Québec, Canada, G7H 2B1, Téléphone : 581-235-5760,

Courriel : virginie.attard1@uqac.ca

\section{Résumé}

Objectifs : La violence sexuelle demeure un problème sociétal touchant tous les groupes sociaux comme les enfants. En ce sens, cette étude évalue un programme de formation à la prévention de la violence sexuelle chez les tout-petits en communautés autochtones. L'étude vise à documenter les effets de la formation au Programme Lanterne|Awacic ainsi qu'à identifier les barrières et les leviers propres à ce type de formation.

Méthode : Menée auprès de 42 intervenants, cette étude évaluative de nature mixte a privilégié l'utilisation de trois types de collecte de données : le questionnaire, l'entrevue et le groupe de discussion.

Résultats: Des effets positifs de la formation ont été observés à très court terme ( $p$. ex., augmentation des connaissances et des croyances). Les résultats sont plus mitigés quant à son effet sur l'utilisation des outils à moyen terme (p. ex., faible utilisation des outils). Les résultats mettent également en exergue l'importance du processus de sécurisation culturelle dans les projets menés dans les communautés autochtones, la nécessité d'impliquer la population locale dans le projet et l'appréciation des participants face à la formation au Programme Lanterne|Awacic.

Implications : La formation permet d'outiller les professionnels œuvrant auprès des enfants d'âge préscolaire à intervenir en matière de prévention de la violence sexuelle. Les résultats suggèrent néanmoins qu'un rappel de la formation aurait permis de maintenir les effets à plus long terme. En somme, les résultats indiquent la pertinence de poursuivre les efforts de prévention dans les communautés autochtones.

Mots-clés: Prévention; violence sexuelle; promotion des relations respectueuses; petite enfance; communautés autochtones; évaluation de programme. 


\section{Introduction}

Depuis une trentaine d'années, la société a pris conscience de l'ampleur des agressions sexuelles (AS) commises envers les enfants. Dans leur méta-analyse, incluant 45 études réalisées dans 24 pays, Barth et ses collègues (2013) estiment que la prévalence de l'AS envers les enfants est de 18-20\% chez les filles et de 8 \% chez les garçons à l'échelle mondiale. Au Québec, en 2006, ce sont 9,7\% des hommes et $22 \%$ des femmes qui rapportent avoir été victimes d'au moins un incident d'AS avec contact avant l'âge de 18 ans, ce qui représente $16 \%$ de la population québécoise (Tourigny et al., 2008). Concernant les communautés autochtones, de $25 \%$ à $50 \%$ des personnes auraient été victimes d'AS dans l'enfance (Collin-Vézina et al., 2009).

L'ampleur et les conséquences associées font du phénomène de l'AS un enjeu social majeur (Tourigny et Baril, 2011). Chez les enfants, les conséquences de l'AS sont pernicieuses et peuvent provoquer, en plus des séquelles physiques, des troubles mentaux comme un état de stress post-traumatique ou encore des problèmes de comportements (Hébert, 2011). Ces conséquences peuvent également persister jusqu'à l'âge adulte (Hailes et al., 2019). En milieu autochtone, non seulement les conséquences associées à l'AS perdurent, mais elles tendent à s'intensifier en raison du contexte socio-économique et politique et de l'éloignement géographique des grands centres urbains (Collin-Vézina et al., 2009; Morin et Lafortune, 2008; Muckle et Dion, 2008). De surcroit, l'encouragement à garder le silence ajoute une dynamique particulière aux communautés autochtones (Morin et Lafortune, 2008). Dans ce contexte, la prévention revêt une importance primordiale. Cette étude porte sur la mise en place et l'évaluation d'un programme de formation destiné aux intervenants en milieux autochtones afin d'accompagner les enfants dans leur développement d'habiletés de protection dans deux communautés autochtones atikamekw.

\section{Les agressions sexuelles en milieu autochtone}

Depuis la colonisation, les peuples autochtones ont été soumis à une déstructuration de leur mode de vie. À partir de 1850, plusieurs lois disparates ont été votées concédant une autorité de l'état colonisateur sur les populations autochtones. Toutefois, l'Acte des Sauvages (Statuts du Canada, 1876), réintitulé par la suite Loi sur les indiens, est le premier acte politique qui vient unifier les lois politiques précédentes. À l'heure actuelle, cette loi est toujours en vigueur et elle est considérée comme ayant une influence majeure sur les difficultés vécues par les Autochtones (Fast et Collin-Vézina, 2019; Morency et Kistabish, 2001). À travers cette loi, diverses mesures d'assimilation ont été mises en place, ce qui a engendré de multiples traumatismes chez les communautés autochtones. En retirant le pouvoir politique des communautés, les autochtones se sont retrouvés soumis à un mode de décision sur lequel ils n'ont eu aucune prise possible. Notamment, la réduction des territoires et le confinement dans des réserves ont engendré un changement draconien dans le mode de vie des Autochtones (Dion et al., 2016; Fast et Collin-Vézina, 2019; Muckle et Dion, 2008; Ross et al., 2016). Bien que I'AS reste une problématique alarmante au sein des communautés, peu de recherches ont été réalisées avec les milieux autochtones (Dion et al., 2018). Pourtant, cette population fait face à des enjeux spécifiques. Au regard de l'éloignement géographique des communautés autochtones des grands centres urbains, il apparait difficile pour les personnes de dénoncer leur agresseur sans crainte de représailles et en toute confidentialité. Par exemple, les déplacements au sein des communautés sont aisément visibles de tous au regard de la faible superficie des territoires. De surcroit, la confidentialité des victimes n'a pas toujours été respectée au sein des services sociaux et des services de santé (Morin et Lafortune, 2008). II semble toujours pénible de parler de violence sexuelle et d'échanger sur le sujet (Morin et Lafortune, 2008). La proximité géographique avec l'agresseur, les sentiments de honte ou de peur ou encore le fait de ne pas vouloir créer de bouleversements intracommunautaires seraient des explications possibles au silence des victimes (Picard, 2004). De plus, une méfiance des Autochtones envers les services publics peut également freiner certaines personnes dans leur dévoilement d'AS (Bergeron et al., 2015). La présence de professionnels allochtones représente donc une entrave supplémentaire à l'accompagnement de personnes victimes d'AS. En effet, les relations entre les allochtones et les Autochtones sont teintées par le passif colonisateur (Conseil Canadien de la Santé, 2012), mais également par les différences culturelles.

\section{La prévention de la violence sexuelle}

Au regard de ce contexte, le Programme Lanterne|Awacic a tenté de répondre à la problématique de la violence sexuelle auprès des enfants en bas âge en formant les professionnels travaillant avec cette population au sein de communautés autochtones. Ce programme s'inscrit dans le contexte politique du dernier plan d'action encadrant la prévention de la violence sexuelle (Gouvernement du Québec, 2016). Depuis 2018, dans le milieu scolaire, les contenus d'éducation à la sexualité sont obligatoires, excepté pour les élèves du préscolaire qui constituent pourtant une population à risque. De surcroit, les jeunes enfants sont ceux qui ont le plus de difficulté à dévoiler une AS (Paine 
et Hansen, 2002).

L'Organisation mondiale de la santé (OMS; 2012) et le Center for Disease Control and Prevention (CDC; Basile et al., 2016) préconisent le développement des programmes de prévention des AS selon le modèle des cercles d'influence qui prend en compte tous les systèmes qui gravitent autour de la personne. Perçue comme une intervention systémique, la prévention doit se faire à plusieurs niveaux, soit individuel, relationnel, communautaire et sociétal. S'attaquer aux facteurs de risque est la meilleure méthode à adopter pour sensibiliser les personnes à la problématique de l'AS. La formation de professionnels gravitant auprès des enfants permettrait donc de reconnaître les signes de violence sexuelle et de connaitre le comportement à adopter face à un dévoilement.

Quatre études ont évalué les programmes de prévention pour les enfants en bas âge : le programme ESPACE (Hébert et al., 2001), Smarter, Safer Kids (Brown, 2017), Parents as teachers of Safety (Kenny, 2009) et Behavioral Skills Training Program (Sarno et Wurtele, 1997). L'évaluation de ces programmes met en exergue que l'apprentissage et la connaissance des parties intimes et de leur dénomination apparaissent comme des facteurs essentiels pour favoriser la réussite des ateliers de prévention. En effet, ces connaissances permettent aux enfants de développer des habiletés de protection et de reconnaître des situations potentiellement à risque ou dangereuses. À titre d'exemple, les situations d'AS sont souvent présentées comme un jeu auprès des enfants (p. ex., jouer au médecin). Toutefois, une acquisition ponctuelle des connaissances n'est pas suffisante, ces acquis ont besoin d'être ancrés dans la mémoire des enfants. L'instauration d'une routine pour les journées de prévention et la répétition des précédentes activités permettent ainsi aux enfants de mieux retenir les informations. C'est pourquoi la planification des ateliers sur plusieurs séances apparait également comme un élément fort des études consultées (Kenny, 2009; Sarno et Wurtele, 1997).

Ces initiatives semblent principalement se concentrer sur les enfants, délaissant parfois la place et le rôle du parent en tant qu'acteur dans la prévention de la violence sexuelle. Pourtant, selon Kenny (2009), l'acquisition des connaissances sera accrue si celles-ci sont réinvesties dans leur cadre familial (p. ex., lors du bain, demander à l'enfant de nommer les parties de son corps). Par ailleurs, les violences commises envers les enfants en bas âge sont généralement perpétrées par un adulte connu de l'enfant, et souvent par un parent ou un membre de la famille élargie (Hébert et Daignault, 2015). Pour les parents, il peut être difficile de s'imaginer qu'un agresseur puisse se retrouver dans leur environnement immédiat. Ainsi, la sensibilisation et l'éducation des parents en matière de prévention de la violence sexuelle apparaissent essentielles. Aussi, il apparait important de former les professionnels qui côtoient les enfants au quotidien comme les intervenants dans les CPE et les professeurs dans les écoles, mais également ceux qui les accompagnent de manière ponctuelle dans les services de santé et les services sociaux.

\section{Les conditions favorables à l'implantation d'un programme}

Les conditions favorables d'implantation d'un programme apparaissent de nature structurelle et organisationnelle. La promotion d'un programme auprès de partenaires, mais aussi de milieux permet de susciter l'intérêt des personnes et ainsi créer une volonté de participation au programme (Minore et Hofner, 2014). Une seconde condition favorable à l'implantation d'un programme réside dans la motivation (Fixsen et al., 2009) et l'engagement des personnes à prendre part aux activités (Fenouillet, 2011). La communication transparente avec les collaborateurs (Minore et Hofner, 2014) et l'importance de transmettre des consignes claires aux partenaires sont des facteurs inhérents à l'implantation d'un programme (Bandura, 1976; Gardner, 2004). La formation de professionnels sur le terrain, dans la pré-implantation d'un programme, revêt une place essentielle dans les conditions favorables puisque cela permet d'outiller les intervenants et d'identifier les situations problématiques (Bergeron et al., 2015; Kirby, 2001; Manseau et al., 2005). Afin de favoriser les effets positifs, la formation doit être encadrée par des animateurs qualifiés et elle doit présenter un contenu de qualité prenant en compte les besoins spécifiques du terrain afin de maintenir une légitimité auprès des collaborateurs (Minore et Hofner, 2014). Former adéquatement les professionnels apparait être un enjeu majeur à l'implantation d'un programme. En ce sens, le Programme Lanterne|Awacic a souhaité en faire l'une de ses priorités. 


\section{Méthodologie}

\section{La formation au programme Lanterne|Awacic}

Pour répondre au manque de programmes de prévention de la violence sexuelle chez les 0-5 ans au sein de communautés autochtones atikamekw, le Programme Lanterne|Awacic ${ }^{1}$ a été développé par la Fondation MarieVincent, en partenariat avec des Atikamekw et le Conseil de la Nation Atikamekw, à la suite d'une analyse des besoins. En effet, les résultats de cette analyse ont révélé que les communautés demandaient à être formées sur cette problématique (Hébert et al., 2017). Une formation (voir Tableau 1) et divers outils ont été créés (voir Tableau 2), en collaboration avec des artistes atikamekw et en se basant également sur les recommandations en matière de prévention de la violence sexuelle auprès des tout-petits (Attard, 2021).

Tableau 1. Description de la formation Lanterne|Awacic

\begin{tabular}{|c|c|}
\hline Caractéristiques & Description \\
\hline Public cible & Toute personne intervenante et éducatrice provenant de milieux atikamekw qui travaille auprès des tout-petits. \\
\hline Durée de la formation & Une journée de 6 heures OU deux demi-journées de 3 heures. \\
\hline Objectifs spécifiques & $\begin{array}{l}\text { 1. Accroître leurs connaissances en matière de violence sexuelle, d'éducation à la sexualité et de promotion des } \\
\text { relations respectueuses }{ }^{2} \text { chez les tout-petits. } \\
\text { 2. Amorcer une réflexion sur les pratiques en matière d'éducation à la sexualité et de promotion des relations } \\
\text { respectueuses auprès des jeunes enfants. } \\
\text { 3. S'approprier les outils du Programme Lanterne|Awacic pour faire davantage de prévention de la violence } \\
\text { sexuelle au quotidien. }\end{array}$ \\
\hline Moyens & $\begin{array}{l}\text { - Présentation de contenu par le biais d'un support Powerpoint } \\
\text { - Partage d'expériences dans le cadre des interventions des professionnels } \\
\text { - Présentation d'outils de prévention }\end{array}$ \\
\hline
\end{tabular}

Objectifs

La présente étude s'appuie sur un devis mixte (quantitatif et qualitatif) afin d'obtenir un point de vue holistique des effets (évaluation sommative) et les facteurs ayant facilité l'implantation (évaluation formative) de la formation au Programme Lanterne|Awacic (Gresham, 2007; McConaughy et Ritter, 2014; Shapiro et Kratochwill, 2000), ainsi qu'à offrir des recommandations pratiques. Les objectifs de l'évaluation sont 1) d'évaluer les effets de la formation sur la modification des croyances, l'acquisition de connaissances et le niveau d'aisance des professionnels à intervenir et à utiliser les outils du programme, 2) documenter la perception des professionnels quant à la pratique sécurisante des animatrices lors de la formation et à la création des outils et 3) d'identifier les barrières et les leviers propres aux formations de prévention en matière de violence sexuelle au sein des communautés autochtones.

\section{Recrutement}

La collecte de données s'est réalisée en trois temps afin de répondre aux volets quantitatifs et qualitatifs de l'étude à l'automne 2019. L'échantillon principal a été collecté par le biais de questionnaires rempli avant et après la formation ( $n=42)$ et deux échantillons secondaires ont été collectés lors d'un groupe de discussion $(n=5)$ une semaine après la formation et d'entrevues téléphoniques $(n=10)$ deux mois après la formation. Lors de chaque volet de l'étude, le consentement libre et éclairé de chaque participant a été obtenu. Une approbation éthique a été obtenue pour réaliser cette étude par le comité institutionnel d'éthique de la recherche avec des êtres humains de l'Université du Québec à Chicoutimi (UQAC) et celui de l'Université du Québec à Montréal (UQAM).

\footnotetext{
1 Pour en savoir plus sur le programme Lanterne, veuillez-vous référer au site suivant : https://cdn.marie-vincent.org/wpcontent/uploads/2019/07/D\%C3\%A9pliant Parents.pdf.

${ }^{2}$ Le terme de relations respectueuses a été favorisé à celui de relations égalitaires par les personnes autochtones travaillant avec la Fondation MarieVincent.
} 
Tableau 2. L'arborescence des outils du programme Lanterne|Awacic

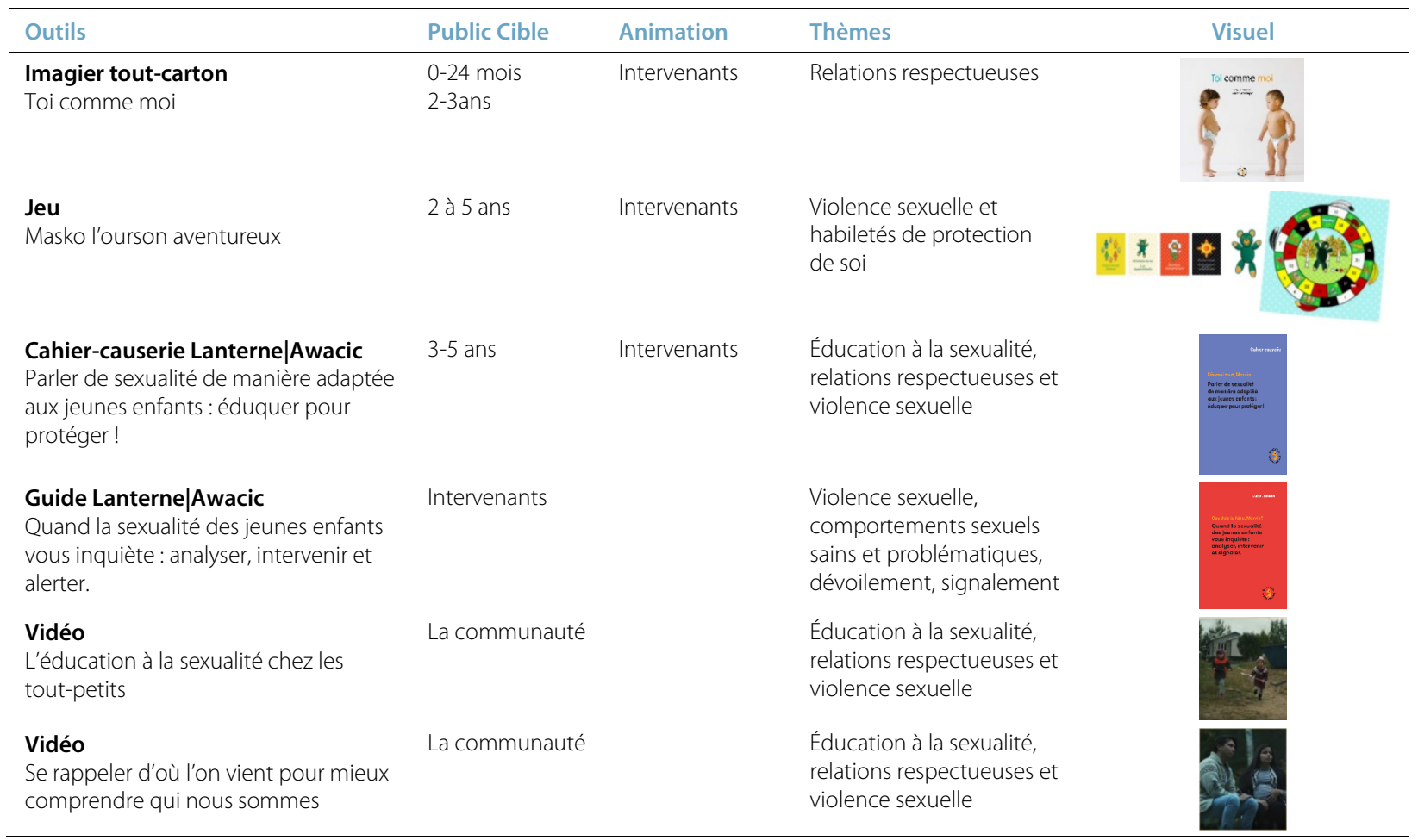

Tableau 3. Le profil des participants de l'étude $(n=42)$

\begin{tabular}{lcc}
\hline Caractéristiques & $\mathrm{N}$ & $\%$ \\
\hline Genre & 38 & 90,5 \\
Femme & 4 & 9,5 \\
Homme & & \\
Âge & 8 & 19,1 \\
$18-29$ ans & 11 & 26,2 \\
$30-39$ ans & 11 & 26,2 \\
$40-49$ ans & 9 & 21,4 \\
$50-59$ ans & 3 & 7,1 \\
60 ans et plus & & \\
Première langue apprise & & \\
Atikamekw & 33 & 78,6 \\
Français & 8 & 19,0 \\
Autres & 1 & 2,4 \\
Domaine d'étude & & \\
Administration & 1 & 2,7 \\
Criminologie & 1 & 2,7 \\
Enseignement & 5 & 13,5 \\
Ergothérapie & 1 & 2,7 \\
Santé & 3 & 8,1 \\
Service de garde & 3 & 8,1 \\
Technique policière & 1 & 2,7 \\
Travail social & 22 & 59,5 \\
Formation passée sur la violence sexuelle & & \\
Non & 28 & 68,3 \\
Oui & 13 & 31,7 \\
Moment de la formation passée & & \\
Moins de 5 ans & & \\
Plus de 5 ans & 9 & 64,3 \\
& 5 & 35,7 \\
\hline
\end{tabular}




\section{Les participants}

Sur les 56 personnes ayant été approchées pour l'étude, 42 ont consenti à y participer, ce qui correspond à un taux de participation de $75 \%$. L'échantillon comprend $90 \%$ de femmes et la moyenne d'âge se situe dans la quarantaine. Au total, $78 \%$ des participants ont comme langue maternelle l'Atikamekw. Au sein des milieux de travail, l'Atikamekw et le français apparaissent comme les deux langues principalement utilisées. L'échantillon est composé de travailleurs sociaux exerçant dans plusieurs milieux comme le CPE (37\%), les écoles (22\%) ou encore les services sociaux (22\%). Concernant la prévention de la violence sexuelle et la promotion des relations respectueuses, $68 \%$ des participants ont affirmé n'avoir jamais reçu une formation sur ce sujet. Ainsi, $32 \%$ des participants certifient qu'ils ont déjà été formés sur la problématique de la violence sexuelle et parmi ces participants, $64 \%$ d'entre eux ont reçu une formation sur cette thématique dans les cinq dernières années.

\section{Les instruments de mesure}

Les outils utilisés pour les collectes de données ont été développés par des personnes allochtones et autochtones, puis soumis à l'expertise de personnes autochtones atikamekw et innu.

\section{Les questionnaires prétest et post-test}

Profil sociodémographique. Lors du prétest, une série de 15 questions ont été posées afin de dresser un profil des participants. Des questions classiques à propos de l'âge, du genre, de la langue maternelle (et parlée au travail) et de l'emploi ont été posées.

Connaissances à l'égard de la violence sexuelle et de la promotion des relations respectueuses. Cette mesure présente aux deux temps de collecte comprend 11 questions pouvant se répondre par Vraiou Faux, dont cinq qui abordent la violence sexuelle et six qui concernent la promotion des relations respectueuses. La valeur accordée à une mauvaise réponse est de « 0 » et « 1 » pour une bonne réponse. Le score total peut varier entre 0 et 11.

Croyances face à la violence sexuelle $(\alpha=, 79)$. Cette section, également présente aux deux temps de mesure, comprend 12 énoncés pouvant être répondus avec une échelle allant de Fortement en désaccord (0) à Fortement en accord (4). Six items représentent des croyances stéréotypées (ces items ont été recodés de manière inversée) et six autres sont exemptes de préjugés. Le score total varie de 0 à 48 et plus le score est élevé, plus leurs croyances sont favorables à l'égard de la prévention de la violence sexuelle, l'éducation à la sexualité et la promotion des relations respectueuses.

Sentiment d'autoefficacité à appliquer et transmettre des connaissances $(a=, 77)$. Cette section remplie au pré et post-test comprend 9 énoncés pouvant être répondus sur une échelle de 0 à 4 , où les participants doivent préciser à quel point ils se sentent capables d'accomplir ce qui est décrit par l'item : Je me sens incapable de pouvoir le faire (0) à Je suis certain de pouvoir le faire (4). Le score total se situe entre 0 et 36; un score plus élevé signifie un meilleur sentiment d'autoefficacité.

Perception d'autoefficacité quant à l'utilisation des outils éducatifs Lanterne|Awacic $(a=, 82)$. Cette section est présente seulement au post-test et évalue la perception d'être capable d'utiliser chacun des quatre outils du Programme Lanterne|Awacic à l'aide d'une échelle en 5 points allant de Je me sens incapable de pouvoir le faire (0) à Je suis certain de pouvoir le faire (4). Le score total varie entre 0 et 16.

Appréciation à l'égard de la formation $(a=, 91)$. Au post-test seulement, les participants ont été invités à répondre à 8 énoncés sur leur appréciation du contenu, des activités, de l'animation ainsi que leur appréciation globale de la formation. Ces items se répondent à l'aide d'une échelle variant entre Fortement en désaccord (0) à Fortement en accord (4) et le score total varie entre 0 à 32.

\section{Le groupe de discussion sous forme de repas communautaire}

Une grille d'entrevue a permis d'aborder les thèmes suivants: I'appréciation générale de la formation, le transfert et l'application des connaissances ainsi que la sécurisation culturelle. Contrairement aux questionnaires, et par manque de temps, la grille du groupe de discussion n'a pas pu être validée par des représentants de communautés autochtones. Avec l'accord verbal des participants, l'entrevue de groupe a été enregistrée par le biais d'un dictaphone et des prises de notes ont été effectuées concernant l'ambiance, les termes récurrents comme celui d'abus sexuel et les recommandations. 


\section{Les entrevues téléphoniques}

La grille des entrevues téléphoniques a été développée en suivant le même processus que celle des questionnaires et a été testée par des personnes autochtones. Les thèmes de cette grille se centraient sur les acquis de la formation sur le moyen terme, la mise en pratique de ceux-ci dans leur travail, leur perception au regard de la présence d'une formatrice autochtone et l'utilisation des outils.

\section{Le traitement et l'analyse des données}

Les analyses descriptives, de même que les Test-t appariés ont été réalisés à l'aide du logiciel SPSS (version 25). Les questions ouvertes du questionnaire de même que les données qualitatives issues du groupe de discussion et des entrevues téléphoniques ont été analysées, à l'aide du logiciel NVivo (version 12), par regroupement thématique d'idées (Miles et Huberman, 1994) et ensuite interprétées à la lumière de l'approche du transfert de connaissance (Graham et al., 2006), des principes directeurs en recherche évaluative autochtone (Chandna et al., 2019) ainsi que des valeurs fondamentales de recherche en milieu autochtone (Lafrance et Nichols, 2008). Par la suite, la triangulation des données qualitatives et quantitatives (Cohen et Manion, 1980) a permis de produire une interprétation plus complète des données quantitatives et qualitatives.

\section{Résultats}

Les résultats sont présentés sous deux rubriques : d'abord celle liée aux effets de la formation, par la suite celle présentant les conditions d'implantation.

\section{Les effets associés à la participation à la formation Lanterne|Awacic}

Les résultats des analyses visant à explorer les effets de la formation sont présentés au Tableau 4.

Tableau 4. Moyennes et écarts-types de chaque instrument et tailles d'effet des différences entre les temps de mesure $(n=42)$

\begin{tabular}{lcccc}
\hline & Prétest & Post-test & Résultat & $\begin{array}{c}\text { Taille } \\
\text { d'effet }\end{array}$ \\
\cline { 2 - 5 } & $\boldsymbol{M}(\boldsymbol{E T})$ & $\boldsymbol{M}(\boldsymbol{E} \boldsymbol{T})$ & $\boldsymbol{t}$ & $\boldsymbol{\eta}^{\mathbf{2}}$ \\
\hline Connaissances - score total (0-11) & $7,51(2,24)$ & $7,56(3,28)$ & $-2,534^{*}$ & 0,15 \\
Violence sexuelle (0-5) & $2,86(1,10)$ & $3,16(1,46)$ & $-3,851^{* * *}$ & 0,29 \\
Relations respectueuses (0-6) & $4,65(1,57)$ & $4,40(2,07)$ & $-0,513$ & $n .5$. \\
Croyances (0-48) & $35,39(5,93)$ & $38,66(7,00)$ & $-4,605^{* * *}$ & 0,38 \\
Sentiment d'autoefficacité (0-36) & $27,69(4,11)$ & $31,73(4,62)$ & $-5,701^{* * *}$ & 0,50 \\
\hline
\end{tabular}

Note. ${ }^{*} p<, 05,{ }^{* *} p<, 01,{ }^{* * *} p<, 001$

\section{Les connaissances}

Les résultats révèlent que la participation à la formation est associée à une augmentation significative des connaissances des participants entre le pré et le post-test, et plus particulièrement pour l'échelle de prévention de la violence sexuelle. Cette augmentation peut être qualifiée de grande lorsqu'on observe la taille d'effet des différences entre les temps de mesure (Cohen, 1988). Aucune augmentation significative des connaissances n'est observée pour l'échelle de promotion des relations égalitaires. Toutefois, les scores obtenus à cette échelle avant la formation étaient déjà élevés pour l'ensemble des participants. De plus, lors des entrevues téléphoniques, deux participants ont mentionné déjà détenir certaines connaissances en la matière.

\section{Les croyances}

La participation à la formation est également associée à une augmentation significative des croyances exemptes de préjugés et favorables à l'égard de la prévention de la violence sexuelle, à l'éducation à la sexualité et à la promotion des relations respectueuses. Selon Cohen (1988), la taille d'effet associée à cette différence peut être qualifiée de grande. Cela se traduit également dans leurs interventions puisque les professionnels ont mentionné parler davantage d'éducation à la sexualité auprès des tout-petits lors de l'entrevue téléphonique. Afin de soutenir leurs interventions auprès des enfants, les participants souhaiteraient que les parents de la communauté bénéficient eux aussi de la formation. 
"[...] Parce que s'ils ne les ont pas, on a beau essayer de leur transmettre nos... les outils qu'on a reçus lors de cette formation-là, si eux [les parents] ils n'ont pas les mêmes informations, ça va être difficile. »

"Parce que même si... même si on les [les parents] a vite amenés suivre la formation, c'est que ça soit donné par nous, il faut qu'il y ait quand même quelqu'un comme vous qui puisse approuver que oui c'est vrai et que la formation est importante aussi, pis les sujets de la formation aussi. »

\section{La perception d'autoefficacité quant à l'utilisation des outils}

De manière générale, les participants ont révélé au post-test être certains de pouvoir utiliser les outils, notamment à $77 \%$ pour le jeu Masko et à $69 \%$ pour le Cahier-Causerie. II est à noter que certains d'entre eux n'intervenaient pas directement auprès d'enfants ce qui a pu influer leur choix de réponse concernant ces items. Durant le groupe de discussion, les participants ont expliqué que les outils présentés étaient intéressants. Ils ont particulièrement apprécié les capsules vidéo, le jeu Masko et le Cahier-Causerie. Deux mois après la formation, bien qu'aucun des participants aux entrevues n'avait encore utilisé le Jeu Masko, ils ont évoqué vouloir l'utiliser prochainement. Concernant les autres outils, ils ont indiqué ne pas les avoir encore utilisés. Ainsi, l'évaluation de l'utilisation des outils n'a pas pu être réalisée après la formation.

\section{La perception d'autoefficacité à appliquer et transmettre des connaissances}

Au regard des résultats liés aux questionnaires, les participants se sentent davantage outillés pour répondre aux questions des enfants en matière de sexualité, de leur enseigner des habiletés de protection contre la violence sexuelle et d'utiliser les vraies dénominations pour parler des organes génitaux et de sexualité. De plus, ces derniers se sentent plus aptes à intervenir auprès d'un enfant en cas de suspicion de violence sexuelle et d'encourager les enfants à se confier à un adulte de confiance. Enfin, ils ont également amélioré leur sentiment d'autoefficacité au regard des stéréotypes et de l'égalité entre les genres. Lors des entrevues téléphoniques, les participantes ont mentionné que la formation leur a également permis d'échanger avec leurs collègues sur les situations problématiques qui les ont mis mal à l'aise, ce qui a été très apprécié.

"Durant la formation, je me suis sentie très à l'aise. J'ai aimé partager mes expériences avec d'autres intervenants. C'est important de se retrouver pour partager les expériences de chacun.»

\section{Application des connaissances dans le milieu professionnel}

Lors du groupe de discussion, les participants mentionnaient le souhait de partager les savoirs acquis avec leurs collègues qui n'avaient pas pu assister à la formation. D'ailleurs, cinq des 10 personnes interrogées lors des entrevues téléphoniques ont indiqué qu'elles avaient échangé avec leurs collègues sur la notion de relations respectueuses entre les enfants ou encore sur les démarches à suivre en cas de dévoilement d'une situation de violence sexuelle.

"J'ai partagé les connaissances que j'ai eu durant la formation avec des amies qui travaillent [intervenants, services sociaux] elles aussi avec des enfants, mais à [nom d'une communauté]. »

La majorité des participants aux entrevues téléphoniques ont expliqué que l'acquisition de connaissances leur avait permis de se sentir plus à l'aise dans leurs interventions. Ils ont également expliqué que l'utilisation des vrais termes pour définir les parties intimes était devenue une de leur priorité dans leurs interventions. Concernant le processus de dévoilement de violence sexuelle, une personne a mentionné qu'elle avait été confrontée à une situation et qu'elle avait retenu qu'il était important de ne pas biaiser le témoignage de l'enfant et de ce fait, elle s'est sentie plus vigilante dans son intervention.

\section{Application des connaissances dans le milieu familial}

Plusieurs participants ont expliqué que les connaissances sur l'éducation à la sexualité, telles que l'existence de plusieurs types d'orientations sexuelles, le développement de l'enfant sur sa sexualité, l'utilisation des vrais termes pour les parties génitales, servaient auprès de leurs enfants et de leurs petits-enfants. Ils utilisent également le livre Toi comme Moi au sein de leur famille, qui leur permet de mettre en lumière les relations respectueuses. 


\section{Les effets associés à la participation à la formation Lanterne|Awacic}

\section{Le processus de sécurisation culturelle}

Les résultats des analyses qualitatives suggèrent que la formation était empreinte de sécurisation culturelle et que l'identité culturelle a été prise en compte dans le programme, ce qui peut faciliter son implantation. La présence d'une formatrice autochtone a été très appréciée, car elle a permis de faciliter les échanges (certaines discussions se sont déroulées en Atikamekw) ainsi que d'instaurer un climat de sécurité culturelle.

\section{"Oui, c'est une bonne idée qu'une Atikamekw fasse partie des formatrices, cela aide à refléter notre identité. »}

D'ailleurs, une des personnes ayant participé aux entrevues téléphoniques a expliqué qu'elle devait se remémorer de parler en français pour la compréhension de la formatrice allochtone. Concernant la formatrice allochtone, un des participants aux entrevues téléphoniques a mentionné que l'important n'est pas l'origine de la personne, mais plutôt les valeurs qu'elle prône et la façon dont elle partage son savoir. Ainsi, la présence d'une formatrice allochtone ne semble pas avoir été un biais aux interactions. Le duo de formatrices a donc été fortement apprécié et a permis aux participants de se sentir libres d'utiliser leur langue maternelle. Le respect des valeurs est également un aspect important mis en exergue par les participants tout comme le savoir-être des formatrices.

Concernant les outils, l'utilisation de couleurs propres à la culture autochtone (noir, blanc, jaune et rouge), la langue et la réalité de ceux-ci semblent avoir rejoint la majorité des participants aux questionnaires. Les vidéos réalisées par et pour des Atikamekw ont également été appréciées puisqu'elles étaient réalisées dans la langue maternelle (avec sous-titres en français pour les professionnels allochtones).

"La formation a été adaptée à un milieu autochtone. Quand je reçois de la formation, il faut parfois que je l'adapte de retour en communauté parce que le contenu ne parle pas aux membres s'il n'est pas adapté. »

Durant la formation, les animatrices ont utilisé la chasse comme métaphore pour expliquer l'importance de la prévention. Les participants ont expliqué que leur culture était bien représentée dans la formation puisque les exemples utilisés étaient toujours en lien avec la réalité de leur communauté.

\section{Le contexte d'implantation de la formation}

Bien que plusieurs participants aient été surpris de la formation et du sujet abordé, ils indiquent avoir rapidement perçu les bénéfices potentiels de celle-ci sur les plans professionnel et familial. On comprend alors que la formation au Programme Lanterne|Awacic semble avoir été implantée sans informer, au préalable, plusieurs intervenants.

"C'est notre directrice qui nous a demandé de participer [...] Je n'étais pas au courant [...] IIs nous ont quasiment obligées, mais moi ça máa fait plaisir. »

Les professionnels ont néanmoins eu l'impression que cette formation allait pouvoir les aider dans leurs interventions.

\section{L'appréciation de la formation au programme Lanterne|Awacic}

De manière générale, les participants ont beaucoup apprécié la formation. Plus de $80 \%$ d'entre eux ont particulièrement apprécié les outils disponibles et ils recommandent fortement la formation à d'autres personnes.

Dans la section «commentaires sur la formation» des questionnaires, les participants ont indiqué que la formation était très intéressante et facile à suivre et qu'une autre formation permettant de faire un rappel serait très appréciée. La formation des autres membres de la communauté a également été proposée afin que chacun d'entre eux puisse bénéficier des mêmes savoirs.

\section{Discussion}

Les objectifs de cette étude étaient de présenter les effets associés à la formation du Programme Lanterne|Awacic en matière de violence sexuelle et de relations respectueuses et de mettre en exergue les barrières et les facilitateurs à l'implantation d'un programme de prévention. Dans l'ensemble, les résultats indiquent que les 
connaissances et les croyances se sont améliorées entre le prétest et le post-test (c.-à-d., avant et après la formation) et que les participants à l'évaluation semblent se sentir plus aptes à prévenir et à intervenir dans des situations de violence sexuelle. Ils ont aussi fortement apprécié la formation et les outils proposés. Cependant, l'utilisation des outils n'a pas pu être évaluée puisqu'ils ne semblent pas avoir être utilisés dans les deux mois suivant la formation.

\section{Les facilitateurs d'implantation d'un programme de prévention de la violence sexuelle en milieu autochtone}

L'analyse des besoins a été l'un des premiers facilitateurs à l'implantation du programme Programme Lanterne|Awacic dans les communautés. Pendant plusieurs mois, des agents de projet se sont rendus sur place et ont réalisé des cercles de partage afin de recenser les besoins de la population cible. Cette façon de procéder a permis de créer un lien avec l'organisme allochtone. Bien entendu, cet organisme travaille majoritairement sur la prévention des AS, donc le sujet du programme était tout de même annoncé. Toutefois, les rencontres ont permis de mettre en exergue la façon dont les intervenants souhaitaient être accompagnés.

La reconnaissance de l'importance de la prévention apparait également comme un facilitateu à l'implantation d'un programme. Dans la formation au Programme Lanterne|Awacic, les professionnels semblent avoir pris conscience de la nécessité d'actions préventives. Sans le soutien et l'implication de ces personnes, le programme en tant que tel ne pourrait donc pas s'ancrer sur le long terme. Dans le processus de sécurisation culturelle, il est également essentiel que les communautés soient impliquées dès le départ dans le projet de recherche dans une logique de partenariat, mais également de transparence vis-à-vis de la communauté (Chandna et al., 2019). Au-delà de la création du programme, l'animation de la formation joue également un rôle important. Plus haut, les aspects positifs de la présence des deux animatrices ont été mentionnés. II semblerait que leur rôle ait eu un impact important sur les effets de la formation tout comme les valeurs qu'elles ont su véhiculer tel que l'ouverture d'esprit, la confiance, l'honnêteté et l'inclusion (Chadna et al., 2019). En ce sens, la sécurisation culturelle est également un facilitateur d'implantation dans un programme de prévention en milieu autochtone.

Dans le cadre de ce projet, une attention particulière a été portée sur les dimensions de ce processus venant respecter les pratiques autochtones. Ce fonctionnement devrait être inhérent à l'implantation de tout projet en milieu autochtone. Cependant, la distance culturelle peut amener un sentiment de frustration et d'incompréhension chez les individus allochtones et autochtones. La marginalisation du savoir autochtone a longtemps été reproduite, notamment au sein des services sociaux et des services de santé (Ramsden, 1993). Les pratiques sécuritaires sont donc importantes afin de travailler en adéquation avec les populations autochtones.

Lors de la formation, deux personnes étaient responsables de l'animation au sein des communautés autochtones. La mixité du duo, une allochtone et une Autochtone, semble avoir été appréciée par la majorité des participants. Dans le processus d'évaluation, il était important de prendre en compte les retombées de ce duo, d'autant plus que la méfiance envers les professionnels allochtones est une réalité, notamment en milieu urbain (DSPCIUSSSMontréal, 2015; Flynn, 2010) et que la problématique touche un sujet tabou (Morin et Lafortune, 2008). Dans le cadre de ce projet, le transfert de connaissances s'exécute par le biais de la formation. Le succès dans ce processus réside dans la prise en compte de la réalité du terrain. Les relations entre les allochtones et les Autochtones découlent de ce contexte, mais également de la culture dominante et de comment celle-ci s'adapte dans le travail des professionnels allochtones avec des cultures dites minoritaires.

Bien que les résultats de l'évaluation révèlent que le processus de sécurisation culturelle a été respecté en ce qui a trait au contenu et à l'animation de la formation, il est important de souligner que cette étude a été réalisée par des personnes allochtones. De surcroit, c'est un organisme allochtone qui, par le biais des subventions, a choisi d'implanter un programme de prévention auprès de deux communautés atikamekw. Malgré qu'une analyse des besoins ait été réalisée en amont, il reste que la demande originelle du projet provient d'un organisme allochtone.

Ainsi, il apparait important d'être vigilant quant aux conditions d'implantation d'un programme de prévention dans un milieu autochtone. Au sein du projet, les membres d'une communauté doivent bénéficier du statut d'acteur au même titre que les autres professionnels œuvrant dans le projet. 


\section{Les barrières d'implantation d'un programme de prévention en violence sexuelle en milieu autochtone}

Comme expliqué plus tôt, pour que l'implantation d'un programme soit optimale, la nation autochtone doit être impliquée dès le début (Chandna et al. 2019). Lors de la collecte de données, il semble que plusieurs personnes ont appris le jour même que la formation allait se donner. Bien entendu, plusieurs hypothèses peuvent être posées. Par exemple, il est possible que les personnes aient été informées en amont de la formation, par exemple, quelques semaines avant sa tenue et que les personnes l'aient tout simplement oublié. Ici, l'implication des communautés n'est pas remise en cause, mais c'est la temporalité concernant la transmission des informations qui est questionnée. On peut alors se demander si ce manque de continuité (ou de rappel) dans la communication des informations, tel que supposé, n'a pas eu d'effets sur la formation. Plus simplement, il est possible que l'information ne se soit pas rendue aux participants. Subséquemment, on peut considérer cet élément comme une barrière à l'implantation du programme.

Un autre élément perçu comme une barrière à l'implantation du programme réside dans le tabou lié à la sexualité présent dans les communautés. En effet, ce dernier a pu teinter certains des effets de la formation. Selon les participants, les discussions autour de la sexualité semblent prohibées et mal venues. Ce facteur a pu être une barrière à l'implantation du programme et avoir pour conséquence de produire un manque de connaissances antérieures à la formation sur l'éducation à la sexualité (p. ex.., connaissance de son corps, développement psychosexuel) et la santé sexuelle. Par exemple, à plusieurs reprises, les participants ont expliqué que ces croyances concernant la sexualité s'appliquaient également aux autres membres de la communauté et notamment les parents. Dans les valeurs fondamentales de Lafrance et Nichols (2008), les programmes de prévention ne doivent pas se concentrer uniquement sur une partie de la population, mais bien sur l'ensemble afin de favoriser le rétablissement de la santé et du bien-être des communautés. Sans une telle approche, les effets associés à la formation pourraient être fortement diminués. On peut donc alors supposer que pour qu'un changement soit effectif celui-ci doit se réaliser à plusieurs niveaux.

Au début du projet, les parents des communautés devaient également participer à une formation. Toutefois, par manque de temps (c.-à-d., échéance du projet, objectifs non réalisables, rapport à produire pour les bailleurs de fonds) et de financement, il a été décidé de ne pas former les parents. Cette tâche a, semble-t-elle, été déléguée à certains professionnels ayant participé à une formation supplémentaire. ${ }^{3}$ Toutefois, cela impute une responsabilité importante aux professionnels qui est celle du transfert de connaissances au sein des communautés. Ainsi, il est intéressant de se questionner sur la façon de former les membres des communautés. En effet, la formation donnée s'est déroulée sur le temps d'une journée et a imposé un rythme assez soutenu avec un contenu très condensé. La question d'un rappel de formation n'a pas été prévue, ce qui remet en question le principe de la formation, des effets désirés sur le moyen terme et des réels bénéfices de la formation comme l'utilisation des outils. De la sorte, les probabilités d'utilisation des connaissances s'amenuisent avec le temps. L'idée de déposer un savoir unique et d'attendre que les communautés reproduisent ce qui leur a été démontré reste tout de même questionnable puisque cela remet en question les principes de sécurisation culturelle (Chandna et al., 2019; Lafrance et Nichols, 2008).

\section{Limites de l'étude}

Le faible échantillon n'a pas permis de réaliser des statistiques plus sophistiquées, en raison d'un manque de puissance statistique pour détecter des effets. De plus, il n'a pas été possible d'évaluer l'utilisation des outils puisque la majorité des participants ont rapporté ne pas avoir eu le temps de le faire lors du suivi téléphonique (c.à-d., deux mois après la formation).

Par ailleurs, selon certains participants, il semblerait que le processus de collecte de données ait été trop imposant (les questionnaires, le groupe de discussion et les entrevues téléphoniques). Ceci est une autre considération à prendre en compte lors de la réalisation d'études, soit la balance entre ce qu'il est nécessaire de collecter comme information, et celle de ne pas surcharger les participants.

Enfin, la difficulté de recueillir la perception réelle des participants à l'égard du programme est une autre limite de cette étude. Certaines personnes ont peut-être été gênées de critiquer plus ouvertement le programme ou de mentionner leurs insatisfactions au regard de la formation. Hot et al. (s.d.), dans leur document de pratiques dans le milieu de l'éducation, expliquent que les comportements non verbaux relatent plus souvent leur pensée que leur discours. Le contact visuel, les questions directes et le besoin de combler les silences sont souvent perçus comme des

\footnotetext{
${ }^{3}$ Intitulée personne lanterne : une personne par communauté ayant suivi une demi-journée de formation supplémentaire, qui n'a pas fait l'objet d'une évaluation.
} 
maladresses pour les populations autochtones. La différence entre les types d'interactions allochtones et autochtones est donc à prendre en compte lors des collectes de données.

\section{Recommandations}

Au regard des éléments proposés précédemment, la prévention des AS apparait essentielle. Les jeunes enfants sont particulièrement vulnérables face à la problématique de la violence sexuelle. L'évaluation des effets des initiatives de prévention offre des éléments importants pour bonifier les pratiques actuelles dans les milieux autochtones. Ainsi, il apparaissait important de proposer des programmes adaptés aux réalités culturelles comme le Programme Lanterne|Awacic. La présente analyse offre des pistes de recommandations quant à l'implantation de programmes similaires.

Avant toute chose, la création d'un réel partenariat avec les communautés autochtones apparait primordiale. L'analyse des besoins est une étape importante qui doit être réalisée avec toutes les précautions nécessaires (p. ex., se renseigner sur les sujets sensibles, la réalité du milieu et la volonté d'implication des membres des communautés). Le partenariat apparait comme un contrat tacite dans lequel chaque partie échange sur les rôles et les implications de chacun. On ne peut pas partir du postulat selon lequel chaque communauté souhaite être impliquée de manière similaire et continue dans le projet. Ainsi, afin de clarifier les attentes de chaque partie, la communication semble être la clé dans l'implantation d'un programme.

Dans un autre ordre d'idées, le programme de prévention, pour qu'il perdure, se doit d'être renouvelé dans le milieu. Comme il a pu être vu, une seule journée de formation ne semble pas suffisante pour que les effets soient ancrés dans la temporalité. II est important de ne pas sous-estimer la capacité des communautés à s'approprier le sujet et à développer de nouvelles initiatives. Toutefois, penser que ces dernières n'ont pas besoin d'un accompagnement plus soutenu après la formation viendrait reproduire des comportements néocolonisateurs. En effet, ce type de formation peut provoquer des changements internes qui doivent être accompagnés et soutenus afin qu'une mutation sociale se réalise.

La violence sexuelle est une problématique très présente au sein des communautés autochtones. Les programmes de prévention en la matière sont d'une importance extrême afin de réduire les risques d'AS. La prévention prend la forme d'une réponse sociale face à un enjeu sociétal. Cependant, la notion de sexualité reste un sujet tabou pour la majorité des Autochtones. II revient alors au chercheur de réaliser son projet avec beaucoup de précautions et de porter une vigilance particulière aux principes de Chandna et al. (2018) et de Lafrance et Nichols (2008) sur les pratiques évaluatives en milieu autochtone. Bien que la Fondation Marie-Vincent ait conçu le Programme Lanterne|Awacic en partenariat, certaines pratiques, sans le vouloir, ont pu avoir un effet colonisateur. L'évaluation révèle des résultats positifs de la formation à très court terme, mais plus mitigés quant à son effet et à l'utilisation des outils à moyen terme au sein des communautés. Un suivi avec les communautés aurait permis de maintenir les effets sur le moyen terme et éventuellement le long terme.

\section{Financement}

La recherche évaluative a été financée par Avenir d'enfants et la Fondation Marie-Vincent.

\section{Conflits d'intérêts}

Les auteurs n'ont aucun conflit d'intérêt à déclarer.

\section{Références}

Attard, V. (2021). Évaluation de la formation au Programme Lanterne/Awacic en matière de prévention de la violence sexuelle en milieu autochtone atikamekw. Mémoire de maîtrise, Université du Québec à Chicoutimi.

Bandura, A. (1980). L'apprentissage social(Vol.83). Éditions Mardaga

Barth, J., Bermetz, L., Heim, E., Trelle, S. et Tonia, T. (2013). The current prevalence of child sexual abuse worldwide: A systematic review and meta-analysis. International Journal of Public Health, 58(3), 469-483. https://doi.org/10.1007/s00038-012-0426-1

Basile, K. C., DeGue, S., Jones, K., Freire, K., Dills, J., Smith, S. G. et Raiford, J. L. (2016). STOP SV: A technical package to prevent sexual violence. National center for injury prevention and control. Centers for Disease Control and Prevention. https://www.cdc.gov/violenceprevention/pdf/SV-Prevention-Technical-Package.pdf 
Prévenir les violences sexuelles en milieu autochtone : Retour sur la formation au Programme Lanterne|Awacic

Bergeron, A., Boileau, A. et Lévesque, C. (2015). Nāniawig Māmawe Nīnawind. Debout et solidaires. Femmes autochtones disparues ou assassinées au Québec. Femmes autochtones du Québec. http://espace.inrs.ca/id/eprint/3580/1/FAQFemmesautochtonesdisparues-jan2016.pdf

Brown, D. M. (2017). Evaluation of Safer, Smarter Kids: Child sexual abuse prevention curriculum for kindergartners. Child and Adolescent Social Work Journal, 34(3), 213-222. https://doi.org/10.1007/s10560-016-0458-0

Chandna, K., Vine, M. M., Snelling, S., Harris, R., Smylie, J. et Manson, H. (2019). Principles, approaches, and methods for evaluation in Indigenous contexts: A grey literature scoping review. Canadian Journal of Program Evaluation, 34(1). https://doi.org/10.3138/cjpe.43050

Cohen, J. (1988). Statistical power analysis for the behavioural sciences. Lawrence Erlbaum Associates.

Cohen, L. et Manion, L. (1980). Research methods in education. Cohen Research Methods in Education.

Collin-Vézina, D., Dion, J. et Trocmé, N. (2009). Sexual abuse in Canadian Aboriginal communities: A broad review of conflicting evidence. Pimatisiwin: A Journal of Aboriginal and Indigenous Community Health, 71), 27-47. http://www.pimatisiwin.com/uploads/July_2009/04CollinDionTrocme.pdf

Conseil canadien de la santé (2012). Empathie, dignité et respect. Créer la sécurisation culturelle pour les Autochtones dans les systèmes de santé en milieu urbain. https://conseilcanadiendelasante.ca/files/Aboriginal_Report_2012_FR_final.pdf

Dion, J., Collin-Vézina, D. et Lavoie, F. (2018). Les violences sexuelles chez les peuples autochtones. Dans S. Bergheul et M. Fernet (dir.), Les violences à caractère sexuel : Représentations sociales, accompagnement, prévention (p.159-181). Presses de I'Université du Québec. https://doi.org/10.2307/j.ctv6zd94v.13

Dion, J., Hains, J., Ross, A. et Collin-Vézina, D. (2016). Pensionnats autochtones: impact intergénérationnel. Enfances, Familles, Générations, (25). https://doi.org/10.7202/1039497ar

Direction de santé publique du CIUSSS du Centre-Est-de-l'île-de-Montréal (DSP-CIUSSS-Montréal) (2015). Un portrait de santé des Autochtones de Montréal.

Fast, E. et Collin-Vézina, D. (2019). Historical trauma, race-based trauma, and resilience of indigenous peoples: A literature review. First Peoples Child \& Family Review, 14(1), 126-136.

Fenouillet, F. (2011). La place du concept de motivation en formation pour adulte. Savoirs, 1(1), 9-46. https://doi.org/10.3917/savo.025.0009

Fixsen, D. L., Blase, K. A., Naoom, S. F. et Wallace, F. (2009). Core implementation components. Research on Social Work Practice, 19(5), 531-540. https://doi.org/10.1177/1049731509335549

Flynn, C. (2010). Femmes autochtones en milieu urbain et violence conjugale: étude exploratoire sur l'expérience et les besoins en matière d'aide psychosociale selon des hommes et des femmes autochtones concernés par cette problématique [mémoire de maîtrise, Université Laval]. https://corpus.ulaval.ca/jspui/handle/20.500.11794/22232

Gardner, H. (2004). Les intelligences multiples : La théorie qui bouleverse nos idées reçues. Éditions RETZ.

Gouvernement du Québec (2016). Stratégie gouvernementale pour prévenir et contrer la violence sexuelle. Gouvernement du Québec. http://www.scf.gouv.qc.ca/fileadmin/Documents/Violences/Brochure_Violences_Sexuelles.pdf

Graham, I. D., Logan, J., Harrison, M. B., Straus, S. E., Tetroe, J., Caswell, W. et Robinson, N. (2006). Lost in knowledge translation: Time for a map? Journal of Continuing Education in the Health Professions, 26(1), 13-24. https://doi.org/10.1002/chp.47

Gresham, F. M. (2007). Response to intervention and emotional and behavioral disorders: Best practices in assessment for intervention. Assessment for Effective Intervention, 32(4), 214-222. https://doi.org/10.1177/15345084070320040301

Hailes, H. P., Yu, R., Danese, A. et Fazel, S. (2019). Long-term outcomes of childhood sexual abuse: an umbrella review. The Lancet Psychiatry, 6(10), 830-839. https://doi.org/10.1016/S2215-0366(19)30286-X

Hébert, M. (2011). Les profils et l'évaluation des enfants victimes d'agression sexuelle. Dans M. Hébert, M. Cyr et M. Tourigny. L'agression sexuelle envers les enfants - Tome 1 (p. 149-204). Presses de l'Université du Québec. https://doi.org/10.2307/j.ctv18ph3jb.7

Hébert, M. et Daignault, I. (2015). Challenges in treatment of sexually abused preschoolers: A pilot study of TF-CBT in Quebec. Sexologies, 24(1), 21-27. https://doi.org/10.1016/j.sexol.2014.09.003

Hébert, M., Lafrenaye-Dugas, A. J. et Tremblay-Perreault, A. (2017). Prévention de l'agression sexuelle. Enjeux identifiés pour l'élaboration de stratégies efficaces auprès d'enfants d'âge préscolaire [Document inédit]. Fondation Marie-Vincent.

Hébert, M., Lavoie, F., Piché, C. et Poitras, M. (2001). Proximate effects of a child sexual abuse prevention program in elementary school children. Child Abuse \& Neglect, 25(4), 505-522. https://doi.org/10.1016/S0145-2134(01)00223-X

Hot, A., Moldoveanu, M., da Silveira, Y. et Mowatt, M. (s. d.). Soutenir la réussite des élèves autochtones adultes: Partie 2 - Vers un développement de nos compétences. Carrefour formation générale aux adultes. http://www.carrefourfga.com/documents/Partie\%202_Vers\%20un\%20developpement\%20de\%20nos\%20competences.pdf 
Kenny, M. C. (2009). Child sexual abuse prevention: Psychoeducational groups for preschoolers and their parents. Journal for Specialists in Group Work, 34(1), 24-42. https://doi.org/10.1080/01933920802600824

Kirby, D. (2001). Emerging answers: Research findings on programs to reduce teen pregnancy (summary). American Journal of Health Education, 32(6), 348-355. https://doi.org/10.1080/19325037.2001.10603497

LaFrance, J. et Nichols, R. (2008). Reframing evaluation: Defining an Indigenous evaluation framework. Canadian Journal of Program Evaluation / Revue canadienne d'évaluation de programme, 23(2), 13-31. https://evaluationcanada.ca/system/files/cjpeentries/23-2-013.pdf

Manseau, H., Blais, M. et Turcotte, M. (2005). L'importance d'une approche qualitative pour faire voir et prévoir les difficultés d'implantation d'un programme de prévention: L'expérience de formation en Centre Jeunesse, entraînement à l'amour propre. Revue de psychoéducation, 37(1), 41-56.

McConaughy, S. H. et Ritter, D. R. (2014). Best practices in multimethod assessment of emotional and behavioral disorders. Dans P. Harrison et A. Thomas (dir.), Best practices in school psychology: Data-based and collaborative decision making (p. 367-390). National Association of School Psychologists.

Miles, M., et Huberman, M. (1994). Qualitative Data Analysis. Sage.

Minore, R., et Hofner, M. C. (2014). Conditions d'implantation dans le canton de Vaud d'un programme de prévention des violences et des comportements abusifs auprès des jeunes. L'exemple de Sortir Ensemble Et Se Respecter. Centre Hospitalier Universitaire Vaudois. http://fcho.ch/sites/default/files/Rapport_interm\%C3\%A9diaire_19_11_2014.pdf

Morency, J. et Kistabish, R. (2001). Intervention en milieu autochtone: comprendre le passé pour mieux agir aujourd'hui. Psychologie Québec, 18(4), 14-18.

Morin, E. et Lafortune, D. (2008). L'agression sexuelle à l'égard des mineurs en territoires nordiques: perceptions des intervenants. Canadian Journal of Community Mental Health, 27(1), 93-110. https://doi.org/10.7870/cjcmh-2008-0007

Muckle, F. et Dion, J. (2008). Les facteurs de résilience et de guérison chez les autochtones victimes d'agression sexuelle. Revue québécoise de psychologie, 29(3), 59-72.

Organisation mondiale de la santé (2006). Guide sur la prévention de la maltraitance des enfants: intervenir et produire des données. https://www.who.int/violence_injury_prevention/publications/violence/child_maltreatment/fr/

Paine, M. L. et Hansen, D. J. (2002). Factors influencing children to self-disclose sexual abuse. Clinical Psychology Review, 22(2), 271295. https://doi.org/10.1016/S0272-7358(01)00091-5

Picard, P. (2004). L'intervention au sein des Premières Nations : La nécessité d'une approche intégrée. Dans Groupe de recherche et d'interventions psychosociales en milieu autochtone [GRIMPA]. 4e colloque de l'association québécoise Plaidoyer-Victimes. https://aqpv.ca/wp-content/uploads/2004_m16.pdf

Ramsden, I. (1993). Cultural safety in nursing education in Aotearoa. Nursing Praxis in New Zealand, 8(3), 4-10.

Ross, A., Dion, J., Gravel, M., Cyr, M. et Maltais, M-E. (2016). Les conséquences associées à la fréquentation des pensionnats autochtones: Mieux comprendre pour mieux orienter la recherche et l'intervention. Dans C. Giraudeau (dir.), Éléments pour une psychologie de la maltraitance (p. 81-106). Publibook.

Sarno, J. A. et Wurtele, S. K. (1997). Effects of a personal safety program on preschoolers' knowledge, skills, and perceptions of child sexual abuse. Child Maltreatment, 2(1), 35-45. https://doi.org/10.1177/1077559597002001004

Shapiro, E. S. et Kratochwill, T. R. (2000) Introduction: Conducting a multidimentional behavioral assessment. Dans E. S. Shapiro et T. R. Kratochwill (dir.), Conducting school-based assessments of child and adolescent behavior (p. 1-20). Guilford Press.

Statuts du Canada. (1876, 12 avril). Chapitre 18 - Acte pour amender et refondre les lois concernant les Sauvages. p. 45-77. https://www.aadnc-aandc.gc.ca/fra/1100100010252/1100100010254

Tourigny, M. et Baril, K. (2011). Les agressions sexuelles durant l'enfance. Dans M. Hébert, M. Cyr et M. Tourigny (dir.). L'agression sexuelle envers les enfants- Tome 1 (p. 7-42). Presses de l'Université du Québec. https://doi.org/10.2307/j.ctv18ph3jb.4

Tourigny, M., Hébert, M., Joly, J., Cyr, M. et Baril, K. (2008). Prevalence and co-occurrence of violence against children in the Quebec population. Australian and New Zealand journal of public health, 32(4), $331-335$. https://doi.org/10.1111/j.1753-6405.2008.00250.x 